\title{
Phocée byzantine et génoise : une croissance urbaine
}

Byzantine and Genovese Phocaea: urban growth

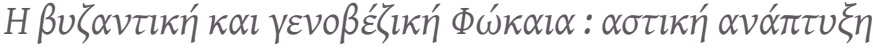

\section{Stephanos Efthymiadis}

\section{QpenEdition}

\section{Journals}

Édition électronique

URL : https://journals.openedition.org/ceb/968

DOI : $10.4000 /$ ceb.968

ISSN : 2261-4184

\section{Éditeur}

INALCO

\section{Édition imprimée}

Date de publication : 9 janvier 2012

ISSN : 0290-7402

\section{Référence électronique}

Stephanos Efthymiadis, «Phocée byzantine et génoise : une croissance urbaine », Cahiers balkaniques [En ligne], 40 | 2012, mis en ligne le 26 mai 2012, consulté le 06 juillet 2021. URL : http:// journals.openedition.org/ceb/968; DOI : https://doi.org/10.4000/ceb.968

Ce document a été généré automatiquement le 6 juillet 2021.

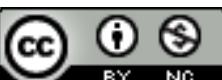

Cahiers balkaniques est mis à disposition selon les termes de la Licence Creative Commons Attribution - Pas d'Utilisation Commerciale 4.0 International. 


\title{
Phocée byzantine et génoise : une croissance urbaine
}

\author{
Byzantine and Genovese Phocaea: urban growth

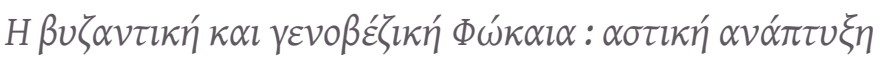

\section{Stephanos Efthymiadis}

1 L'intérêt scientifique que nous avons porté à Phocée est né de la publication du dossier perdu et retrouvé de Félix Sartiaux, qui contient des rapports et des lettres concernant ses fouilles à l'intérieur de la ville et dans ses alentours pendant la période troublée entre 1914 et 1922. C'est en l'an 2000 que mon collègue Andréas Mazarakis, spécialisé dans l'étude de la période de la domination génoise en mer Égée, a découvert dans les archives de l'École Française d'Athènes ce que les archéologues qui ont repris l'œuvre de Sartiaux dans cette région cherchaient depuis longtemps. À coup sûr, le dossier de Sartiaux, que l'on a publié par la suite dans le Bulletin du Centre des études d'Asie Mineure et qui comprend des lettres, des notes d'ensemble, des cartes et des photos, n'offrait qu'un intérêt marginal pour cette période très tardive. Son orientation archéologique le conduisait plutôt vers l'époque archaïque et classique. Ce dossier contenait néanmoins quelques témoignages intéressants sur l'époque byzantine et génoise qui, comme des pièces éparses, pourraient servir à améliorer notre connaissance sur une période de longue durée, mais que l'on qualifie, non sans raison, d'obscure ${ }^{1}$.

2 À la ville ionienne la plus septentrionale, l'Histoire n'a réservé que deux périodes d'apogée économique et sociale : a) la période archaïque où Phocée s'est avérée une métropole et une puissance navale ( $\mathrm{VII}^{\mathrm{e}}$ et $\mathrm{VI}^{\mathrm{e}}$ siècles avant notre ère) et $\mathrm{b}$ ) la période entre le dernier quart du XIII ${ }^{\mathrm{e}}$ siècle et l'an 1455, lorsque la ville a été conquise par les Ottomans. Entre ces deux périodes très éloignées dans le temps, Phocée avait connu le déclin pendant l'époque classique, hellénistique et romaine. D'abord alliée aux Athéniens, elle a été ensuite soumise aux Perses, puis à Alexandre, aux Séleucides, à Attale de Pergame, et enfin aux Romains. Cependant, elle n'est pas passée complètement inaperçue dans les sources qui, outre son passé glorieux, mentionnent, quoiqu'en passant, ce que sa terre et ses habitants produisent: de la céramique, du marbre, des vêtements de pourpre, de verres à boire, des éventails, et surtout des 
richesses minérales, dont la plus importante était la pierre phocéenne utilisée pour les couches, c'est-à-dire pour le pavé des rues ; elle reste en usage même à nos jours²

3 En dépit de son déclin et de sa marginalisation géographique, Phocée n'était pas une ville de dimensions médiocres. Les fouilles de Sartiaux et de ses successeurs turcs ont révélé que le périmètre de sa muraille extérieure était d'un rayon de $5 \mathrm{~km}$. Cela veut dire que Phocée s'étendait bien au-delà de la surface de son promontoire qui, d'ailleurs, lui fournissait la réputation d'être une ville à deux ports. Quelle que soit la direction du vent, le promontoire lui assurait la protection des bateaux qui y naviguaient.

4 L'historien latin Tite-Live appelait ce promontoire «lingua» (XXXVII, 31) et les témoignages des Phocéens, réfugiés en Grèce après 1922, confirment ce que Sartiaux rapporte dans son exposé. La ville disposait de deux portes, une qui donnait sur le petit port et l'hinterland, et une autre qui donnait sur le grand port. À l'époque ancienne, ces deux portes conduisaient les Phocéens de l'acropole protégée d'une muraille solide à sa ville basse. D'après la Chronique catalane de Ramon Muntaner, datée de la première moitié du XIV ${ }^{e}$ siècle, Phocée était divisée en deux parties: le château (lo castell) où habitaient 3 mille Grecs (los grecs) et la ville (la vila), c'est-à-dire la ville basse ${ }^{3}$.

Cette division reflète sûrement une réalité qui resta inchangée au cours du temps, mais qui, pour la période byzantine, ne peut pas être attestée qu'en se rapportant à des sources antérieures ou postérieures. Les historiens byzantins du $\mathrm{VI}^{\mathrm{e}}$ siècle, tels que Procope ou Agathias, ne connaissent Phocée qu'en tant que métropole de Marseille et un même silence règne sur ce qui suit. Rien n'est enregistré sur les invasions perses et arabes, ni sur les raids des pirates durant les $\mathrm{VII}^{\mathrm{e}}$ et $\mathrm{VIII}^{\mathrm{e}}$ siècles.

En ce temps-là Phocée n'est qu'une petite ville qui, gérée par un évêque, s'affichait dans l'administration ecclésiastique. Les évêques de Phocée qui figurent dans les actes des conciles œcuméniques de 691/2 et de 787 constituent des indices d'une certaine croissance démographique. Mais la ville ne semble pas avoir eu une importance dans la géographie chrétienne. Aucun centre de pèlerinage dans la région n'est mentionné dans les sources. Dans les ruines d'une église dédiée aux Taxiarques (les Archanges) qui se situait sur la péninsule phocéenne, Sartiaux a découvert deux ampullae (eulogies) dont l'une portait l'inscription "Apostolos " (Apôtre) et l'autre "hagios Andréas " (Saint-André) ${ }^{4}$. Ces petites bouteilles en forme de gourde devraient être originaires d'un centre de pèlerinage de Saint André, mais faute de tout autre renseignement, on ne peut rien y ajouter. Cela dit, quand en 1308 les Catalans envahissent et pillent la ville, ils emportent une pièce du bois de la Croix que Saint-Jean, lui-même, avait décrochée, la tunique (un camis) sans couture que la Vierge avait cousue pour le disciple bien-aimé de son Fils et, enfin, un manuscrit de l'Apocalypse copié, selon la légende, par Saint-Jean et décoré de pierres précieuses. Nous puisons cette information dans la Chronique de Ramon Muntaner qui ajoute que, lorsque les Turcs ont occupé Éphèse, ils avaient apporté ces reliques à Phocée, comme butin, pour les échanger contre du blés.

7 Pendant la période de l'Antiquité tardive, Phocée n'est connue que pour son type particulier de céramique, surtout celui des vases que l'on appelle Phocaean red-slip ware. À en juger par de pareilles trouvailles dans presque tout le territoire de l'Empire romain d'Orient, il avait existé un réseau commercial au départ de cette ville portuaire. Cela, bien sûr, ne démontre que le lieu de provenance de la matière première, sans indiquer pour autant que Phocée fut nécessairement le lieu de production. Les 
trouvailles de la période suivante montrent que ce commerce perd son dynamisme en se limitant dans le voisinage de la région de Smyrne.

Pourtant, une autre activité artisanale et commerciale paraît avoir été présente. Phocée, semble-t-il, était fameuse pour ses vêtements de pourpre. La source qui nous renseigne à ce sujet n'est pas byzantine, mais occidentale. Il s'agit du récit de la Translation des reliques de Saint Mercure à la ville de Benevento en Italie méridionale. À l'initiative du roi lombard local, le sanctuaire de l'église de Sainte Sophie fut décoré avec des vêtements de pourpre cousus en fil phocéen (de purpureis gausapis... et telis Phocaico stagmine textis) ${ }^{6}$. Cette référence rejoint celle, plus ancienne, des Métamorphoses d'Ovide où on trouve la mention de la pourpre phocéenne (VI, v. 9). Mais pourquoi a-t-elle gardée sa réputation à travers les siècles ? Faut-il supposer un traitement spécial par l'emploi de l'alun, la matière minérale que, quelques siècles plus tard, les Génois allaient exploiter dans un cadre commercial plus global ?

C'est sûrement grâce à son port naturel que la ville n'a pas perdu toute son importance pendant la période médiobyzantine (VIII ${ }^{\mathrm{e}} \mathrm{XII}{ }^{\mathrm{e}}$ siècles.). Située entre Lesbos et Chios, elle offrait un abri aux navigateurs, comme une porte sur la mer pour l'intérieur de l'Asie Mineure. Les quelques voyageurs occidentaux qui ont consigné leurs mémoires par écrit ne lui prêtent pas grande attention. Il faut se tourner vers un texte hagiographique du $\mathrm{XII}^{\mathrm{e}}$ siècle, la Vie du patriarche Léontios de Jérusalem (1110/1115-1185), pour trouver des informations d'une certaine valeur pour l'état de la ville à cette époque. En route vers Constantinople le saint stationne sur une île en face de Phocée avec quelques moines qui l'accompagnent. Un habitant de la ville d'origine amalfitaine, appelé Mauro, invite le saint chez lui et reçoit sa bénédiction grâce à laquelle sa femme a pu concevoir et accoucher d'un enfant, une fille qui a pris le nom de Leontồ ${ }^{7}$.

$10 \mathrm{Vu}$ l'époque et l'endroit, l'installation d'un étranger comme Mauro sur la côte ne doit pas surprendre. À partir de la fin du $\mathrm{XI}^{\mathrm{e}}$ siècle, le développement du commerce naval était géré par les Italiens qui progressivement allaient coloniser le littoral byzantin. Bien qu'on ignore quel était l'objet de l'activité commerciale de Mauro, on ne peut pas exclure qu'elle ait eu un rapport avec l'exploitation de l'alun. Il est vrai que jusqu'au XIII ${ }^{e}$ siècle l'Occident s'approvisionnait en alun, matière essentielle pour la teinture des vêtements, en Égypte. Les Occidentaux qui jusqu'alors s'occupaient de ce commerce étaient les Vénitiens et les Amalfitains, pas encore les Génois.

11 Quant à ces derniers, leur situation a bien changé après 1204. Contrairement à Venise qui a joué un rôle tout à fait négatif envers les Byzantins avant et lors de la conquête de Constantinople en 1204, Gênes ne s'est pas mêlée à l'entreprise de la quatrième croisade et de la démolition de l'Empire byzantin. Une fois la domination byzantine restaurée à Constantinople en 1261 et jusqu'à la fin de l'empire, les Génois deviendront, d'une part, les alliés permanents des Byzantins et, d'autre part, une puissance qui pouvait créer un équilibre avec leur grand adversaire commercial, Venise ${ }^{8}$. C'est dans cette idée d'alliance que, peu après son installation au palais impérial de Constantinople, l'empereur Michel VII Paléologue a généreusement octroyé des privilèges économiques à des familles génoises en échange du paiement de certains impôts. Dans un document qui date de l'an 1268, les frères Zaccaria, Manuele et Benedetto, se portent les gérants du trafic de l'alun, fait qui permet de déduire qu'ils étaient déjà les maîtres de Phocée et de la bande littorale qui allait jusqu'à Adramyttion (Edremit) ${ }^{9}$. Phocée, l'on a déjà insinué, était importante pour ses mines situées dans les montagnes voisines, riches en 


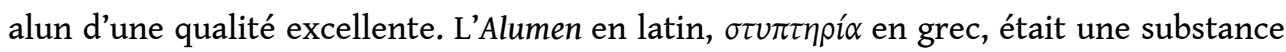
indispensable pour l'industrie textile de l'époque et son exploitation suffisait à maintenir une large entreprise et un trafic maritime de longue distance ${ }^{10}$.

Cette concession de la part de l'empereur byzantin n'était que la suite d'une série d'actes identiques par lesquels des familles génoises devenaient, en fait, les cogouverneurs des possessions du territoire byzantin, tant des îles du nord-est de la mer Égée, que de quelques villes maritimes au long du littoral asiatique. Ces concessions avaient la forme d'un double contrat d'ordre financier. L'empereur byzantin demeurait en titre le propriétaire de ces territoires tandis que Gênes acquérait le droit de les exploiter ainsi que l'obligation de payer comme taxe une somme d'argent à Constantinople. Dans ces accords politiques, Gênes était représentée par des familles d'influence qui géraient les affaires de la république en outremer pour leur compte, mais qui se référaient et restaient dépendantes de la métropole. La concession était ainsi faite ad personam, ce qui veut dire sans droit héréditaire. Tout renouvellement du contrat exigeait la rédaction d'un nouvel acte impérial ${ }^{11}$.

Les Zaccaria de Castro étaient une famille originaire du castello de Gênes et qui possédait une fortune considérable aux environs de la ville-métropole. Les deux frères, Manuele et Benedetto, collaboraient sous forme d'autorisations mutuelles dans le but d'élargir leurs champs d'activité commerciale soit vers la mer Noire soit vers les voies maritimes de l'Occident. Jusqu'à la mort de Benedetto en 1307 ils s'entendaient bien et réglaient leurs entreprises en tirant grand profit. Manuele géra les affaires du Levant, et Benedetto s'occupa de la vente de l'alun et d'autres produits orientaux en l'Occident. Déjà, en 1268, leurs noms apparaissaient dans un document à propos de la livraison d'une cargaison à Aigues-Mortes, port situé à l'embouchure du Rhône. Vers l'an 1281, leur réseau commercial s'étendait sur Majorque, les Flandres et l'Angleterre. De l'exploitation de l'alun jusqu'à la livraison aux teintureries de vêtements s'interposait une série de métiers dont quelques-uns sont mentionnés dans des actes génois. À part les mineurs que l'on suppose être des autochtones, il y avait des calafati (réparateurs de bateaux), des ferrarii (forgerons), des magistri antelami (des architectes de bateaux), des remolarii (fabricants de rames), candelerii (chandeliers), barrilarii (tonneriers), calegarii (cordonniers), etc. Tous venaient travailler à Phocée par contrat signé par un notaire spécial envoyé sur place par l'administration centrale de Gênes ${ }^{12}$.

14 La demande croissante en matières premières, ainsi qu'un service maritime plus rapide et fréquent, fournit une explication satisfaisante à la fondation d'une nouvelle ville près de Phocée. À une distance de $15 \mathrm{~km}$ au nord de la ville qui serait désormais appelée

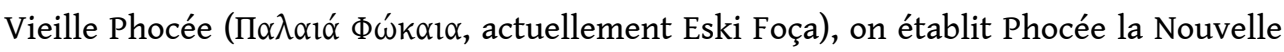

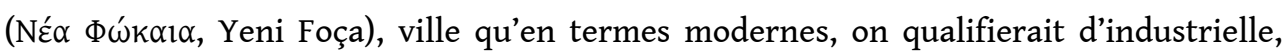
munie d'un port et peuplée par les ouvriers des mines. À en juger par la plus ancienne référence fournie par l'historien empereur Jean Cantacuzène et datant de l'an 1329, la fondation de Phocée la Nouvelle doit avoir eu lieu peu avant cette date, mais toujours dans les années $1320^{13}$.

Grâce à l'exploitation et au commerce de l'alun, maintenu pendant presque deux siècles, Phocée est devenue une ville d'une importance centrale dans le monde méditerranéen. Vu que la puissance du centre byzantin s'affaiblissait de plus en plus, la population perdit sa dépendance vis-à-vis de Constantinople et préféra être en bons termes avec les seigneurs génois. En fait, les habitants de cette région - le littoral de Smyrne à Adramyttion et les îles voisines de la mer Égée - se trouvaient encadrés par 
trois mondes différents. En premier lieu, l'Empire déchu des Romains d'Orient (c'est-àdire les Byzantins), qui, même s'il ne pouvait plus leur assurer une identité politique, représentait leur adhésion à la foi orthodoxe; en second lieu, les Génois, seigneurs complètement intégrés à leur société ; et, en troisième lieu, les Ottomans, qui n'étaient pas toujours considérés comme une menace et avec lesquels, au moins avant la chute de Constantinople en 1453, ils maintenaient des relations commerciales et d'autres natures également.

Cette coexistence est bien mise en évidence dans les écrits de deux historiens du XV ${ }^{\mathrm{e}}$ siècle qui étaient originaires de Phocée ou qui y ont passé une grande partie de leur vie. Le premier et le plus connu est l'historien Doucas, le célèbre auteur d'une œuvre que l'on appelle habituellement Histoire byzantino-turque et qui donne le récit le plus circonstancié du siège final de Constantinople. Pendant une longue période de sa vie que l'on ne connaît pas avec précision, Doucas a été secrétaire au service des seigneurs génois. Dans son histoire, il intègre cette double identité qui porte les traces d'une vie dans un monde qui réunissait les Byzantins et les Occidentaux. Doucas pleure à larmes chaudes la perte de Constantinople et, en même temps, il ne cache pas ses croyances religieuses qui, sous l'influence du monde où il a vécu, sont tout à fait prolatines ${ }^{14}$.

Mais, à part Doucas, il y avait un autre érudit local versé dans l'étude de l'histoire. Il portait un nom d'origine, apparemment, italienne, Jean Canaboutzès, et le titre de magister, qui veut dire maitre ès lettres. Parmi les œuvres qu'il nous a léguées, on doit signaler un traité sur Denys d'Halicarnasse, historien grec de l'époque romaine (vers 60-7 avant notre ère). Dans ce texte, l'auteur veut montrer les liens entretenus entre les Grecs et les Romains d'autrefois, liens qu'il voulait souligner aussi à propos de la vie commune des Romains d'Orient avec les Romains d'Occident, voire les Génois. Outre cela, ce qui est remarquable dans ce traité, c'est sa «terminologie géographique »: à plusieurs reprises, à côté du nom grec classique, qu'il utilise pour désigner une ville ou une localité quelconque, il n'hésite pas à citer le nom grec « vulgaire » de son temps, en $\mathrm{y}$ ajoutant parfois le latin ou le turc ${ }^{15}$. Bref, en tant qu'érudit qui dans son œuvre reflète la langue et la réalité géographique de son époque, Jean Canaboutzès représente une nouvelle mentalité qui, sans doute, dérive de sa propre expérience. Il cherchait à rapprocher ses compatriotes grecs de leurs maîtres génois, sans ignorer le fait que les Ottomans étaient une nouvelle réalité historique dans la région.

En fait, juste avant la chute de deux Phocées en 1455, un Phocéen propose ou représente un hybride d'identité politique et ethnique qui repose sur la floraison économique de sa patrie et sur ce qu'il croyait être la réalité d'un monde qui sortait du modèle byzantin sans espoir de retour. On dirait que cette Phocée était alors devenue une métropole levantine jouissant d'un capitalisme primitif ou naissant qui toutefois n'allait pas survivre à la conquête ottomane. De nouveau, Phocée allait, dans l'histoire, tomber dans l'oubli pour longtemps. 


\section{BIBLIOGRAPHIE}

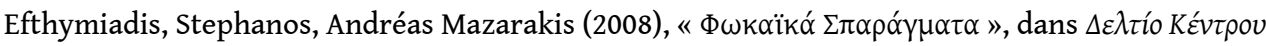

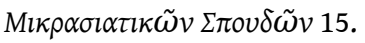

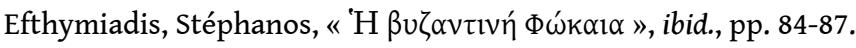

Efthymiadis, Stéphanos, Andréas Mazarakis, (2009) « La chronique familiale du Parisinus gr. 1601 et l'identité de son rédacteur », Byzantinische Zeitschrift 102/2, pp. 615-625.

Flusin, Bernard, (2006), « Prédictions et prophéties dans l'œuvre de Doucas », L'écriture de la mémoire. La littérarité de l'historiographie. Actes du III colloque international philologique Nicosie, 6-7-8 mai 2004, sous la dir. de Paolo Odorico, Panagiotis A. Agapitos, Martin Hinterberger, 6, Paris : Centre d'études byzantines néo-helléniques et sud-est européennes, École des Hautes Études en Sciences Sociales, pp. 353-373.

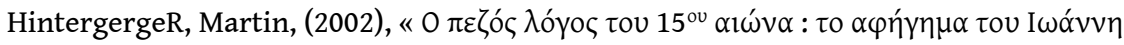

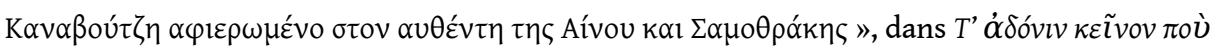
$\gamma \lambda v \kappa \grave{\alpha} \theta \lambda_{\imath} \beta \tilde{\alpha} \tau \alpha$ l. Proceedings of the 4th International Congress Neograeca Medii Aevi (Novembre 1997, Nicosie), sous la dir. de Panagiotis A Agapitos et Michalis Pieris, Iraklion- Crète, pp. 405-425 .

Kotzabassi, Sofia, (2006), « Ist der Kopist des Geschichtswerkes von Dukas Dukas selbst ?», Byzantinische Zeitschrift 96 (2003), p. 679-683 ; Flusin B., « Prédictions et prophéties dans l'œuvre de Doucas », L'écriture de la mémoire. La littérarité de l'historiographie. Actes du III colloque international philologique Nicosie, 6-7-8 mai 2004, sous la dir. de Paolo Odorico, Panagiotis A. Agapitos, Martin Hinterberger, Paris : Centre d'études byzantines néo-helléniques et sud-est européennes, École des Hautes Études en Sciences Sociales, p. 353-373.

Mazarakis, Andréas, (2006), Zaccaria e della Volta nell'Egeo Orientale, 1268-1329, Athènes : Circolo Numismatico ligure « Corrado Astengo », Sezione della Società ligura di Storia patria.

\section{NOTES}

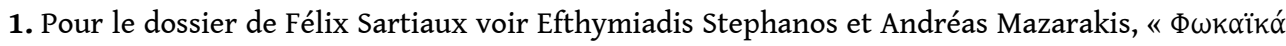

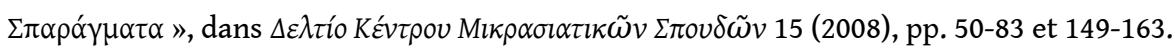

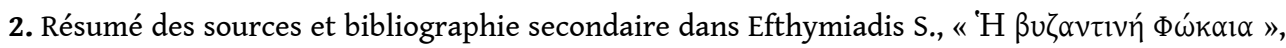
ibid., pp. 84-87.

3. Ramon Muntaner, 1951, Crónica. Text $i$ notes per E. B. (E. Bagué), Barcelone ch. 234, pp. 92-93 ; trad. française par Jean-Marie Barberà dans Ramon Muntaner, 2002, Les almograves : l'expédition des Catalans en Orient, Toulouse, p. 129.

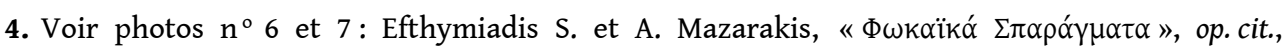
pp. 155-156.

5. Voir Ramon Muntaner, Crónica, op.cit., ch. 293, p. 93 ; trad. française, Les almograves, p. 130.

6. Voir Translatio s. Mercurii, dans Monumenta Germaniae Historica, SS. Rerum langobardicarum et italicarum saec. VI-IX, Hannover 1878, p. 577.

7. Voir The Life of Leontios Patriarch of Jerusalem, ch. 55-56, éd. D. Tsougarakis, 1993, The Medieval Mediterranean 2, Leiden, pp. 94-96.

8. Voir Balard Michel, La Romanie génoise (XII ${ }^{e}$-début du XV siècle), vol. I., Gênes-Rome, 1968, pp. 17-104. 


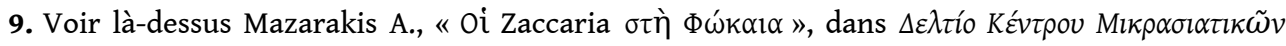
$\Sigma \pi 0 v \delta \tilde{\omega} v 15$ (2008), pp. 105-130.

10. L'étude la plus récente sur ce sujet est celle de Jacoby D., «Production et commerce de l'alun oriental en Méditerranée, $\mathrm{XI}^{\mathrm{e}}-\mathrm{XV}^{\mathrm{e}}$ siècles ", in L'alun de Méditerranée, Philippe Borgard, Jean Pierre Brun et Maurice Picon (dir), Naples/Aix-en-Provence : Collection du Centre Jean Bérard, 23, 2005, p. 219-267.

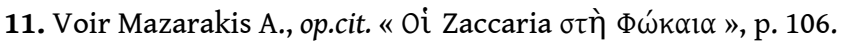

12. L'histoire de cette famille fut retracée par Mazarakis Andréas, Zaccaria e della Volta nell'Egeo Orientale, 1268-1329, Circolo Numismatico ligure "Corrado Astengo ", Athènes, Sezione della Società ligura di Storia patria, 2006.

13. Sur la fondation de Phocée la Nouvelle voir en dernier lieu, Mazarakis A., Zaccaria e della Volta nell'Egeo Orientale, pp. 59-64 et plus précisément p. 63.

14. Sur cet historien, on consultera, en dernier lieu, Kotzabassi, S. «Ist der Kopist des Geschichtswerkes von Dukas Dukas selbst?», Byzantinische Zeitschrift 96 (2003), pp. 679-683; et Flusin B., "Prédictions et prophéties dans l'œuvre de Doucas ", L'écriture de la mémoire. La littérarité de l'historiographie. Actes du III ${ }^{\mathrm{e}}$ colloque international philologique Nicosie, 6-7-8 mai 2004, sous la dir. de P. Odorico, Panagiotis A. Agapitos, Martin Hinterberger, Paris, Centre d'études byzantines néo-helléniques et sud-est européennes, École des Hautes Études en Sciences Sociales, 2006, pp. 353-373.

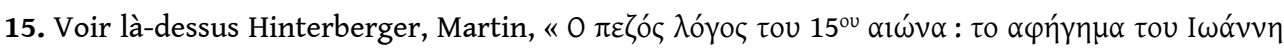

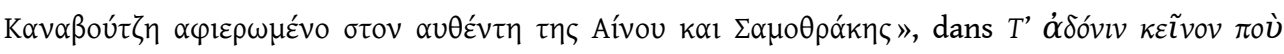
$\gamma \lambda v \kappa \grave{\alpha} \theta \lambda_{\imath} \beta \tilde{\alpha} \tau \alpha_{l}$. Proceedings of the 4th International Congress Neograeca Medii Aevi (Novembre 1997, Nicosie), sous la dir. de Panagiotis A. Agapitos et Michalis Pieris, 2002, Iraklion, Crète, pp. 405-425 ; et, plus récemment, S. Efthymiadis et A. Mazarakis, «La chronique familiale du Parisinus gr. 1601 et l'identité de son rédacteur", Byzantinische Zeitschrift 102/2 (2009), pp. 615-625.

\section{RÉSUMÉS}

La découverte du dossier Sartiaux à l'École Française d'Athènes contribua à compléter nos connaissances sur la Phocée byzantine et génoise. Au XIII ${ }^{e}$ siècle. l'exploitation de l'alun, et sa vente dans tout l'Occident méditerranéen par les Génois, entraînent une forte croissance et la fondation dans les années 1290 de la nouvelle Phocée, un nouveau port «industriel ». Cette prospérité s'accentue dans le siècle suivant et au début du XV ${ }^{\mathrm{e}}$ siècle, la cité est au confluent de trois pouvoirs, l'Empire romain d'Orient moribond, les Génois, seigneurs complètement intégrés à leur société et les Ottomans avec lesquels, au moins avant la chute de Constantinople, ils maintenaient des relations commerciales.

The discovery of the Sartiaux record at the French School of Athens, added to our knowledge of Byzantine and Genovese Phocaea. In the $13^{\text {th }}$ century, the exploitation of alun, as well as its sale throughout the Western Mediterranean by the Genoese led to strong growth and the foundation of the New Phocaea, in the 1290s, which was a new «industrial » port. Prosperity increased by the next century and in the early $15^{\text {th }}$ century, the city is found at the confluence of three distinct powers, the dying Eastern Roma Empire, the Genoese lords, completely integrated into their 
society and the Ottomans, with whom the city maintained commercial relations, at least shortly before the fall of Constantinople.

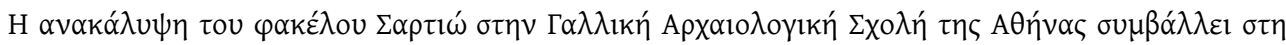

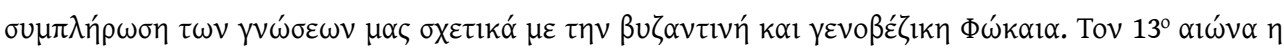

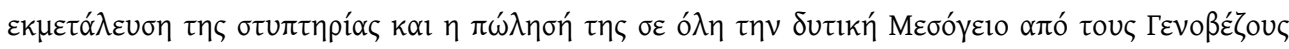

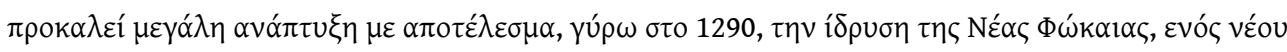

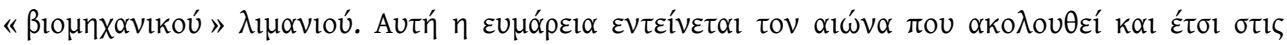

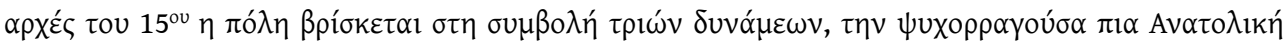

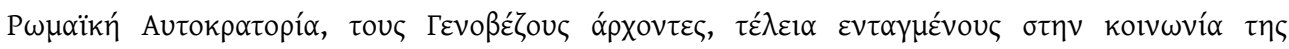

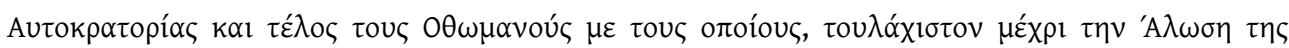

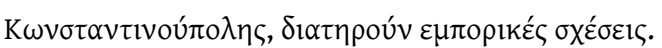

\section{INDEX}

motsclesmk ЏЕНОВА, МАКЕДОНИЈА

Mots-clés : Ampullae/Eulogies, Muntaner Ramon (Chronique), Sartiaux Félix (1876-1944), commerce maritime

motsclestr Türk milliyetçiliği, Foça, İyonya, Anadolu, Cenova, Edremit

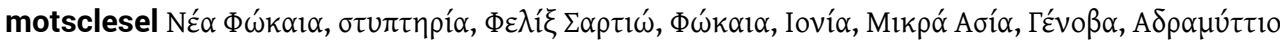
Thèmes : Histoire

Index chronologique : treizième siècle, Empire byzantin

Index géographique : Phocée, Asie Mineure, Edremit, Ionie

Keywords : Phocaea, thirteenth century, Asia Minor, Sartiaux Felix, Byzantine empire, Edremit 